\title{
RNA interference-mediated gene silencing of cyclophilin A enhances the radiosensitivity of PAa human lung adenocarcinoma cells in vitro
}

\author{
XIN JIANG $^{1-3}$, QIAO-LI ZHANG ${ }^{2,4}$, YE-HONG TIAN ${ }^{5}$, JIN-CHANG HUANG ${ }^{2,3}$ and GUO-LIN MA ${ }^{6}$
}

${ }^{1}$ Graduate School, Beijing University of Chinese Medicine; ${ }^{2}$ Department of Acupuncture and Minimally Invasive Oncology, Beijing University of Chinese Medicine Third Affiliated Hospital; ${ }^{3}$ Department of Oncology, Combination Traditional Chinese and Western Medicine, China-Japan Friendship Hospital, Beijing 100029; ${ }^{4}$ Department of Traditional Chinese Medicine, Beijing Hepingli Hospital, Beijing 100013; ${ }^{5}$ Department of Gastroenterology, Xianyang Hospital of Yan'an University, Xianyang, Shanxi 712000; ${ }^{6}$ Department of Radiology, China-Japan Friendship Hospital, Beijing 100029, P.R. China

Received November 11, 2015; Accepted November 21, 2016

DOI: $10.3892 / 01.2017 .5667$

\begin{abstract}
Radiotherapy is currently the major therapeutic strategy for patients with lung cancer. However, radioresistance and various side effects continue to present challenging issues for this form of treatment. A recent study demonstrated that cyclophilin A (CyPA) was overexpressed in non-small cell lung cancer and, therefore, presents a novel potential therapeutic target. In addition, gene-radiotherapy is a novel method for cancer treatment. Therefore, the objective of the present study was to investigate the potential effect of CyPA silencing on radiosensitivity in human lung adenocarcinoma in vitro. The stable CyPA-silencing lung adenocarcinoma (PAa) cell line was generated using lentivirus-mediated small hairpin RNAs. The knockdown of CyPA was determined using fluorescent microscopy and western blot analysis. Cells were irradiated using various doses of cobalt-60 $(0,2$, 4, 6 and $8 \mathrm{~Gy}$ ). The radiosensitizing effects were determined by a clonogenic survival assay. Apoptosis and cell cycle distribution were evaluated using flow cytometry. Silencing of CyPA significantly increased the apoptosis of PAa cells. In addition, the radiosensitivity of cells was markedly enhanced following CyPA silencing. Furthermore, silencing of CyPA, in combination with irradiation, induced $\mathrm{G}_{2} / \mathrm{M}$ phase cell cycle arrest. Taken together, the data suggest that
\end{abstract}

Correspondence to: Dr Jin-Chang Huang, Department of Acupuncture and Minimally Invasive Oncology, Beijing University of Chinese Medicine Third Affiliated Hospital, 51 Xiaoguan Street, Beijing 100029, P.R. China

E-mail: zryhhuang@163.com

Dr Guo-Lin Ma, Department of Radiology, China-Japan Friendship Hospital, 2 Yinghua Dongjie, Beijing 100029, P.R. China

E-mail: maguolin1007@qq.com

Key words: cyclophilin A, RNA interference, lung adenocarcinoma, radiosensitivity the silencing of CyPA, combined with radiation therapy, may increase the therapeutic efficacy of lung cancer treatment through regulation of the cell cycle and apoptosis-associated signaling pathways.

\section{Introduction}

Lung cancer is one of the most common types of malignant tumor, with a rising global incidence. It is currently the most lethal form of malignant tumor globally, with a low five-year survival rate (1). Furthermore, the number of lung cancer cases diagnosed annually is increasing, of which non-small cell lung cancer (NSCLC) accounts for $\sim 80 \%$ (2). Adenocarcinoma is the most common histological subtype of NSCLC (3). Although previous studies have contributed considerably to the understanding of this type of carcinoma, the development of effective targeted therapies is required (3).

In patients who are diagnosed with NSCLC, radiotherapy is one of the primary treatment options (4). However, the radioresistance of lung cancer remains a significant therapeutic obstacle (5). Improving tumor radiosensitivity is an effective way to increase the potency of radiotherapy (6). The combination of gene therapy and conventional radiation is a promising approach in cancer treatment (7).

The ubiquitously distributed protein cyclophilin A (CyPA), also known as peptidylprolyl isomerase $\mathrm{A}$, is a member of the immunophilin family. CyPA possesses peptidyl prolyl cis-trans isomerase (PPIase) activity, and is involved in numerous biological processes including $\mathrm{T}$-cell activation, protein folding, trafficking and molecular chaperoning (8). Previous studies have demonstrated that CyPA is overexpressed in numerous types of cancer, and is an important factor in malignant transformation and metastasis $(9,10)$. The role of CyPA in lung cancer has recently been the subject of various studies. Campa et al (11) demonstrated that CyPA protein levels were significantly raised in lung cancer tissue specimens, compared with adjacent non-diseased lung tissues. Subsequent studies revealed that the suppression of CyPA expression diminishes NSCLC tumor growth through the 
regulation of matrix metallopeptidase $9(12,13)$. However, the role of CyPA in the radiosensitivity of lung adenocarcinoma remains to be elucidated.

A previous study demonstrated that CyPA protein expression in the tissue of lung adenocarcinoma tumors is significantly upregulated following radiation therapy (14). Therefore, in order to further investigate the underlying mechanisms of CyPA gene radiosensitivity in lung adenocarcinoma cells, the current study utilized lentiviral vectors packaged by virus particles to specifically silence the CyPA gene.

\section{Materials and methods}

Materials and reagents. PAa lung adenocarcinoma cells were obtained from Peking University Health Science Center (Beijing, China), and the 293FT human embryonic kidney cell line was purchased from Invitrogen (Thermo Fisher Scientific, Inc., Waltham, MA, USA). Lentiviral vectors [pLLU2G-green fluorescent protein (GFP)], packaging systems (3rd generation lentivirus packing system) and negative control virus particles (pLP1, pLP2, pLP/VSV-G and pLLU2G) were obtained from Invitrogen (Thermo Fisher Scientific, Inc.) Lipofectamine ${ }^{\circledR}$ 2000 transfection reagent and One Shot ${ }^{\circledR}$ Stbl3 $^{\mathrm{TM}}$ chemically competent $E$. coli were purchased from Invitrogen (Thermo Fisher Scientific, Inc.). The QIAquick Gel Extraction Kit was purchased from Tiangen Biotech Co., Ltd. (Beijing, China). Dulbecco's modified Eagle's medium (DMEM), fetal bovine serum (FBS) and diethylpyrocarbonate were all purchased from Sigma-Aldrich (Merck Millipore, Darmstadt, Germany).

Construction of CyPA RNA interference (RNAi) lentivirus vector. For the silencing of CyPA expression, DNA oligonucleotides were designed based on the CyPA siRNA sequence (5'-TCTCGAGTTTTTCTCGAGA-3'), and cloned into pLLU2G lentiviral vectors to construct pLLU2G-CyPA small hairpin (sh)RNA plasmids, according to the method reported previously (15). Briefly, DNA oligonucleotides were ligated with plasmid pLLU2G and digested with $\mathrm{HpaI}$ and XhoI to form double stranded DNA. The double stranded DNA was then transformed into One Shot Stbl3 ${ }^{\mathrm{TM}}$ Chemically Competent $E$. coli (Thermo Fisher Scientific, Inc.). Negative control virus particles (pLP1, pLP2, pLP/VSV-G and pLLU2G) from Invitrogen (Thermo Fisher Scientific, Inc.) were used to monitor the nonspecific reactions induced by the shRNA, and to optimize the efficiency of virus transduction according to the manufacturer's protocol.

Viral packaging. Lentiviral vectors were produced by the transient transfection of 293T cells, as described previously (16). The 293FT cells $\left(\sim 5 \times 10^{6}\right.$ cells) in logarithmic growth phase were inoculated into $10 \mathrm{~cm}$ culture dishes and cultured for $24 \mathrm{~h}$ in a humidified $5 \% \mathrm{CO}_{2}$ atmosphere at $37^{\circ} \mathrm{C}$. The vectors were subsequently transfected into the $293 \mathrm{FT}$ cells using Lipofectamine ${ }^{\circledR} 2000$ and incubated overnight under the same conditions. The following day, DMEM containing $10 \%$ FBS was changed and the viral supernatants were collected following $48 \mathrm{~h}$ under the same conditions, filtered using $0.45 \mu \mathrm{m}$ pore size filters and stored at $-80^{\circ} \mathrm{C}$. For the determination of infectious titers, 293FT cells were infected with lentivirus (CyPA shRNA and Control shRNA) (dilution,
1:10) and incubated overnight at $37^{\circ} \mathrm{C}$ with $5 \% \mathrm{CO}_{2}$. The cells were subsequently washed in PBS and cultured for an additional $48 \mathrm{~h}$ under the same conditions. GFP-positive cells were counted using a BD FACSVerse ${ }^{\mathrm{TM}}$ flow cytometer and BD FACSuite software (version 1.0) (both BD Biosciences, Franklin Lakes, NJ, USA).

Transduction of PAa lung adenocarcinoma cells. PAa lung adenocarcinoma cells were inoculated into 6-well plates $\left(1 \times 10^{5}\right.$ cells/well) and divided into three groups, including blank (no transfection), negative control (transduction of the pLLU2G-eGFP plasmid) and CyPA-siRNA (pLLU2G-CyPA-EGFP). Three replicates were performed for each group. GFP expression was detected via fluorescence microscopy (Nikon Corporation, Tokyo, Japan) to determine the infection efficiency. The protein expression of CyPA was detected by western blot analysis.

Western blot analysis of CyPA. Total cellular protein was extracted using an M-PER Mammalian protein extraction kit (Thermo Fisher Scientific, Inc.). Total protein $(25 \mu \mathrm{g})$ was then separated by SDS-PAGE on a $15 \%$ gel and transferred to a polyvinylidene difluoride membrane. The membrane was blocked with $5 \%$ non-fat milk in Tris-buffered saline with Tween 20 (TBST) for $1 \mathrm{~h}$ at $4^{\circ} \mathrm{C}$, and incubated overnight at $4^{\circ} \mathrm{C}$ with the primary antibody directed against CyPA $(1: 1,000$ dilution; cat. no. ab126738) or $\beta$-actin (1:2,000 dilution; cat. no. AM1021B) (both Abgent Biotech Co., Ltd.). Following washing 3 times with TBST, the membrane was incubated with a corresponding horseradish peroxidase-conjugated goat anti-rabbit IgG (1: 7,500 dilution; cat. no. 111-035-144; Jackson ImmunoResearch, Inc., Chicago, USA) for $1 \mathrm{~h}$ at $4^{\circ} \mathrm{C}$. Protein bands were detected using the Pierce ECL Western Blotting Substrate Kit (Thermo Fisher, Scientific, Inc.), followed by exposing the membrane to Hyperfilm on the Kodak Digital Science $^{\mathrm{TM}}$ Image Station 440CF (both Kodak, Rochester, NY, USA). This analysis was repeated 3 times. Quantitative analysis of CyPA protein expression was conducted using Image J software (version 1.38e; National Institutes of Health, Bethesda, MD, USA) ( $\mathrm{n}=3$; mean \pm standard deviation).

Clonogenic survival assay. Lung adenocarcinoma cell suspensions in the logarithmic growth phase were inoculated into $25 \mathrm{~mm}^{2}$ culture bottles at various densities $(600,1,000,4,000$, 8,000 and 10,000 cells). The blank group, negative control group and CyPA-siRNA group were each irradiated with doses of $0,2,4,6$ and 8 Gy (dose rate, $1 \mathrm{~Gy} / \mathrm{min}$ ) at the ${ }^{60} \mathrm{Co}$ Radiation Center of Beijing Normal University (Beijing, China). The experiments were repeated three times and duplicates were set up for each group.

Following irradiation, the cells were placed in a $37^{\circ} \mathrm{C}$ incubator and cultured for 10 days to form clones. The colonies were fixed by treatment with $100 \%$ methanol for $5 \mathrm{~min}$ and subsequently stained with $0.5 \%$ crystal violet for $5 \mathrm{~min}$ at room temperature. Colonies of PAa cells exposed to various radiation doses in each group were counted using Gel-Pro Analyzer software (version 4.0; Media Cybernetics, Inc., Rockville, MD, USA). Colonies with $>50$ cells were counted as colony-forming units. The clone formation rate (\%) was calculated according to the following formula: (Number 

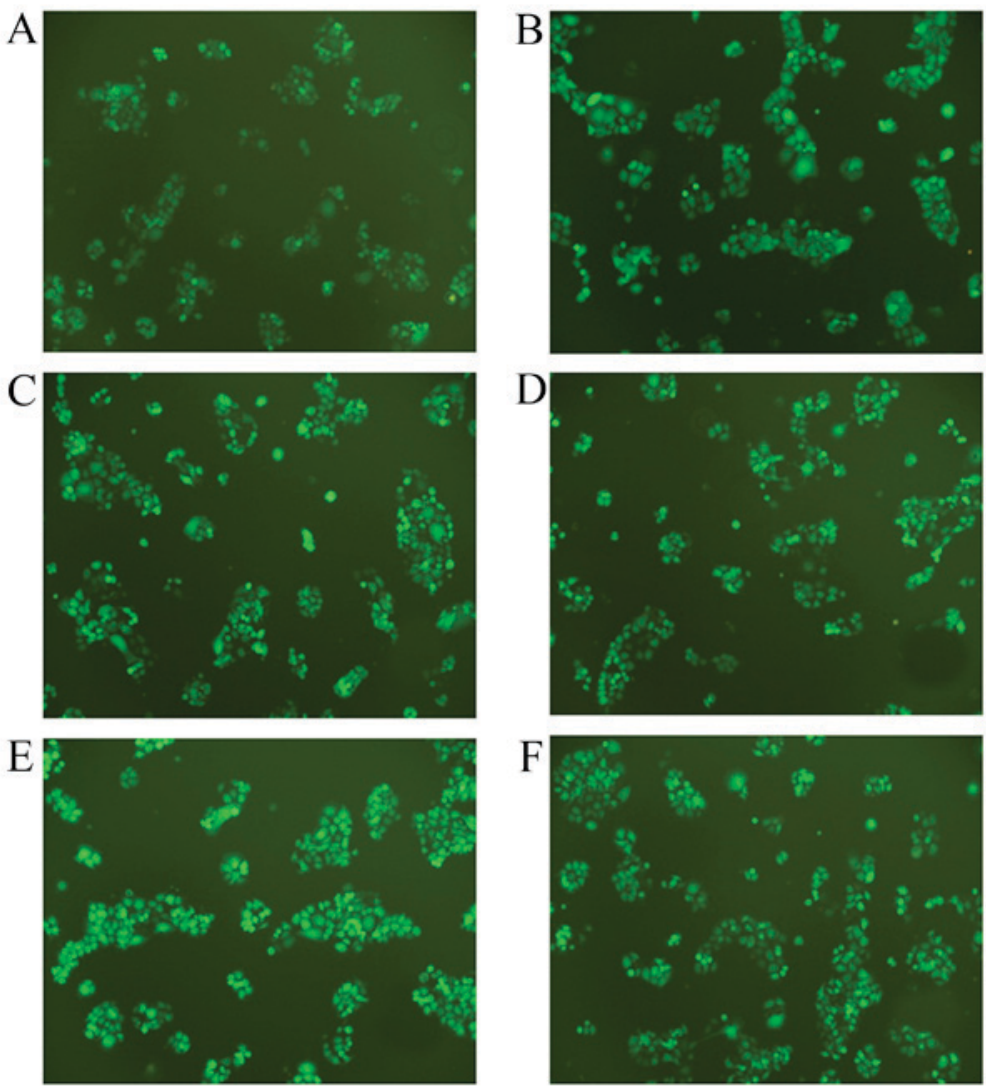

Figure 1. Fluorescence analysis of PAa cells with lentiviral vector transfection. (A) Blank group (PAa cells without transduction). (B) Negative control group (transduction of pLLU2G-eGFP plasmid). (C) CyPA-shRNA-1 (transduction of pLLU2G-CyPA-eGFP). (D) CyPA-shRNA-2. (E) CyPA-shRNA-3. (F) CyPA-shRNA-4. Duration of incubation, 48 h. Magnification, x100. PAa, cyclophilin A-silencing lung adenocarcinoma; CyPA, cyclophilin A; eGFP, enhanced green fluorescent protein; shRNA, small hairpin RNA.

of colonies formed/number of cells seeded) $\mathrm{x} 100$. The cell survival fraction (SF) was calculated as follows: (Number of colonies formed following treatment/number of cells seeded) $\mathrm{x}$ inoculation efficiency. The inoculation (plate) efficiency was calculated as follows: Number of colonies counted/number of cells inoculated. The multi-target single-hit model was used to evaluate the radiation sensitivity of CyPA-siRNA cells. $\mathrm{D}_{0}$ is the mean lethal dose resulting from the multi-target model. $\mathrm{D}_{\mathrm{q}}$ is defined as the quasithreshold dose.

Cell cycle and apoptosis analysis by flow cytometry. Lung adenocarcinoma cells $\left(1.5 \times 10^{6} / \mathrm{ml}\right)$ in the logarithmic growth phase were suspended in RPMI-1640 medium supplemented with $10 \%$ fetal calf serum (Sigma-Aldrich; Merck Millipore) and inoculated into $25 \mathrm{~mm}^{2}$ culture bottles. A total of three groups were set up as aforementioned. Following adherent growth for $24 \mathrm{~h}$, the cells were exposed to ${ }^{60} \mathrm{Co}$ radiation of 0 or 2 Gy. Following radiation, the cells were continuously cultured for $24 \mathrm{~h}$ at $37^{\circ} \mathrm{C}$. For each radiation dose, 3 parallel experimental bottles were established and the experiment was repeated 3 times.

The cells were harvested $24 \mathrm{~h}$ following radiation and centrifuged at $200 \times \mathrm{g}$ for $5 \mathrm{~min}$ at room temperature. The supernatant was discarded, the cells were washed with PBS twice and then fixed with cold anhydrous ethanol at $4^{\circ} \mathrm{C}$ for $12 \mathrm{~h}$. Cells were then filtered with 400 mesh nylon filters and washed with PBS twice. Finally, the cells were incubated with
$10 \mathrm{ng} / \mathrm{ml}$ RNase A and $500 \mu \mathrm{g} / \mathrm{ml}$ propidium iodide on ice for $30 \mathrm{~min}$, and analyzed with the FACScan ${ }^{\mathrm{TM}}$ System (BD Biosciences). Cell cycle phases were analyzed using CellQuest $^{\mathrm{TM}}$ software (version 5.7; BD Biosciences).

Statistical analysis. Statistically significant differences between the groups were determined using the Student's $t$-test (two groups) or one-way analysis of variance, followed by a post hoc Dunnett's T3 test (multiple groups). The data were analyzed using SPSS version 20.0 (IBM SPSS, Armonk, NY, USA). $\mathrm{P}<0.05$ was considered to indicate a statistically significant difference.

\section{Results}

Expression analysis of CyPA in stably transfected cell lines. To silence the CyPA gene, CyPA-specific shRNA plasmids were constructed and transfected into PAa cells. A total of four plasmids, termed shRNA-1, shRNA-2, shRNA-3 and shRNA-4, were used. Fig. 1 presents the stable transfected cell lines under fluorescence microscopy, with a relatively high transfection efficiency being achieved.

To further determine the silencing of the CyPA gene, western blot analysis of protein expression in the stable transfected PAa cell lines was performed. $\beta$-actin was used as the internal control. As presented in Fig. 2A, the expression of CyPA was low or undetectable in the cell lines 
A

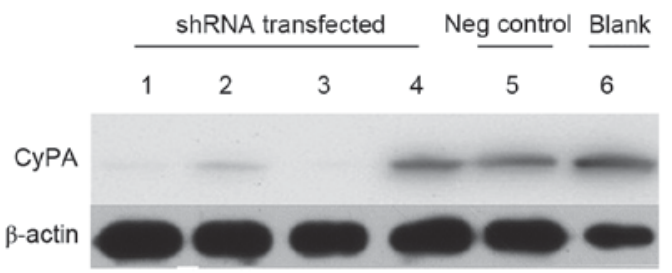

B

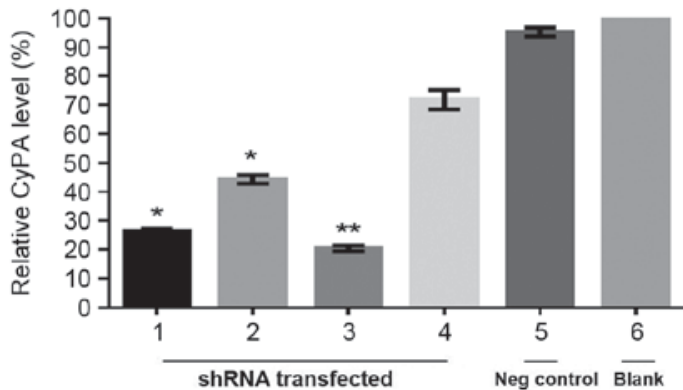

Figure 2. Knockdown of CyPA expression in the transfected PAa cell line. (A) Representative western blot of CyPA expression levels in PAa cell lines with and without CyPA-shRNA transfection. $\beta$-actin was used as an internal loading control. (B) Relative expression of CyPA. Protein levels of CyPA were normalized to $\beta$-actin and the data were expressed as the percentage of the blank control. $\mathrm{N}=3$ (mean \pm standard deviation). ${ }^{*} \mathrm{P}<0.05$ and ${ }^{* *} \mathrm{P}<0.01$, compared with the blank group. Lane 1, CyPA-shRNA-1; lane 2, CyPA-shRNA-2; lane 3, CyPA-shRNA-3; lane 4, CyPA-shRNA-4; lane 5, Negative control (Transfection with the vector without CyPA shRNA); lane 6 , Blank (PAa cell line without transfection). PAa, cyclophilin A-silencing lung adenocarcinoma; CyPA, cyclophilin A; shRNA, small hairpin RNA; Neg, negative.

transfected with CyPA-shRNA-1, -2 and -3 compared with the blank group, indicating an effective knockdown of the CyPA gene. CyPA expression was then normalized to the internal control and the relative expression levels were quantitatively analyzed (Fig. 2B). The protein expression of CyPA in the cell line transfected with CyPA-shRNA-1, -2 and -3 was significantly lower, compared with the blank group without transfection (CyPA-shRNA-1, P=0.0085; CyPA-shRNA-2, $\mathrm{P}=0.035$; CyPA-shRNA-3, $\mathrm{P}=0.024)$. There was no significant difference between the CyPA-shRNA-4 group and the blank group ( $\mathrm{P}=0.257)$. Among the three effective shRNA vectors (shRNA-1, -2 and -3), shRNA-3 resulted in relatively higher transfection efficiency and lower CyPA expression levels (Fig. 2B). In the shRNA-3 group, CyPA protein expression was knocked down by $85 \%$ compared with the blank group. Therefore, the cell line transfected with the CyPA shRNA-3 vector was utilized for the subsequent experiments.

Silencing of CyPA gene significantly enhances the apoptosis of PAa cells. To investigate the effect of CyPA silencing on lung cell growth without radiation, the in vitro apoptosis of PAa cells treated with CyPA shRNA-3 was analyzed using flow cytometry. During the culture period, few apoptotic cells $(<10 \%)$ were present in the blank and negative control groups (Fig. 3). By contrast, a significant increase in apoptotic damage was observed in CyPA-silenced cells, with an average cell apoptosis rate of $55.5 \%(\mathrm{P}=0.0145)$.

CyPA gene silencing enhances the radiosensitivity of PAa cells. The effect of CyPA silencing on the sensitivity of PAa cells to

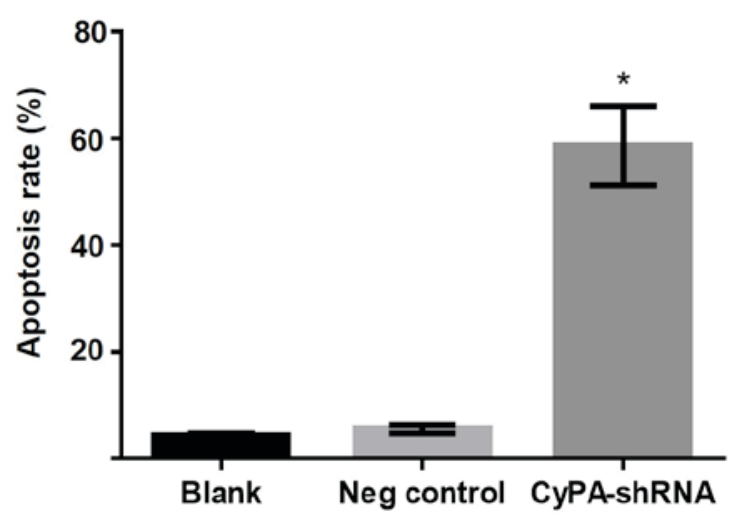

Figure 3. Apoptosis of cyclophilin A-silencing lung adenocarcinoma cells is enhanced by CyPA silencing. Data are expressed as the mean \pm standard deviation $(n=6) .{ }^{*} \mathrm{P}<0.05$, compared with the blank group. shRNA, small hairpin RNA; CyPA, cyclophilin A; Neg, negative.

radiation was subsequently assessed using a clonogenic cell survival assay. Irradiation resulted in a marked dose-dependent apoptotic effect in PAa cells (Fig. 4). The number of colonies decreased with the increase in radiation dose for all three groups. The CyPA-silenced cell line exhibited a significantly decreased survival fraction compared with the blank group at 2 Gy, indicating greater radiosensitivity ( $P=0.0308$; Fig. 4B). Multi-target single-hit model fitting survival curves provided a sensitization enhancement ratio of $1.157 \pm 0.005$.

Increased cell cycle arrest at the $G_{2} / M$ phase occurs in CyPA-silenced PAa cells prior to and following radiation. Cell cycle analysis using flow cytometry was performed to determine whether CyPA gene silencing affects the cell cycle distribution of PAa cells prior to radiation. The representative cell cycle distribution is presented in Fig. 5A. No significant difference was observed in $G_{0} / G_{1}$ and $S$ phase cells among the three groups (Fig. 5B). However, the number of cells at the $\mathrm{G}_{2} / \mathrm{M}$ phase was significantly increased in the CyPA-silenced group, compared with the control group $(\mathrm{P}=0.035)$.

To investigate whether CyPA silencing has a greater effect on the cell cycle distribution of PAa cells post-radiation, the cell cycle distribution was analyzed following irradiation with ${ }^{60} \mathrm{Co}$ at $2 \mathrm{~Gy}$. Significantly fewer cells were at the $\mathrm{G}_{0} / \mathrm{G}_{1}$ and $\mathrm{S}$ phase in the CyPA-silenced group, compared with the blank and control groups (all $\mathrm{P}<0.05$; Fig. 6). Following radiation at $2 \mathrm{~Gy}$, the percentage of $\mathrm{G}_{2} / \mathrm{M}$ phase cells in the CyPA-silenced group was $32.15 \pm 0.86$, whereas only $18.37 \pm 0.15 \%$ of blank group cells were in the $\mathrm{G}_{2} / \mathrm{M}$ phase.

\section{Discussion}

Since the development of RNAi in animals, personalized gene therapy utilizing this technique has been evaluated in various human diseases, with $>2,000$ clinical trials implementing RNAi-mediated gene therapy completed or ongoing worldwide by 2013 (17). During these trials, engineered viruses were often exploited to efficiently deliver therapeutic genes to the target cells (17). In the present study, the selection of shRNA sequences was evaluated based on the effective silencing of 
A

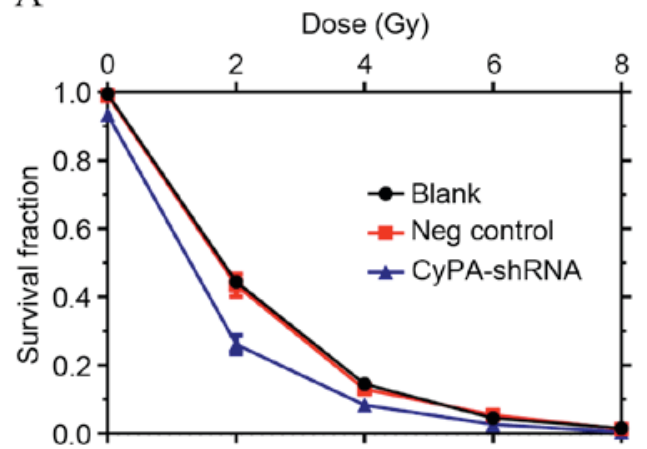

B

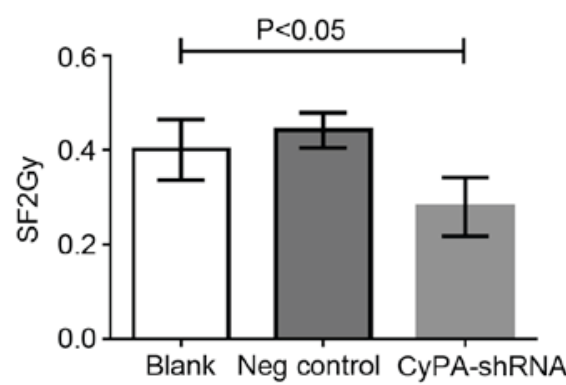

Figure 4. CyPA silencing enhances the radiosensitivity of PAa lung cancer cells. (A) The decrease in the survival fraction of PAa lung cancer cells correlated with increasing radiation dose. (B) The significant reduction of the cell survival fraction in the CyPA silencing group compared with blank controls, at 2 Gy. PAa lung cancer cells were irradiated at various doses $(0,2,4,6$ and $8 \mathrm{~Gy})$. Following radiation, the cells were cultured for 10 days to form clones. The cell survival fraction was calculated as: (Number of colonies formed following treatment/number of cells seeded) $\mathrm{x}$ inoculation efficiency. SF2 Gy indicates the survival fraction of PAa cells at a dose of 2 Gy. N=6 for each group and each radiation dose. CyPA, cyclophilin A; PAa, cyclophilin A-silencing lung adenocarcinoma; shRNA, small hairpin RNA; Neg, negative.
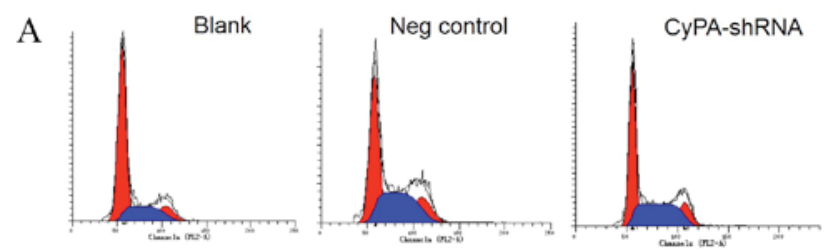

$\mathrm{B}$

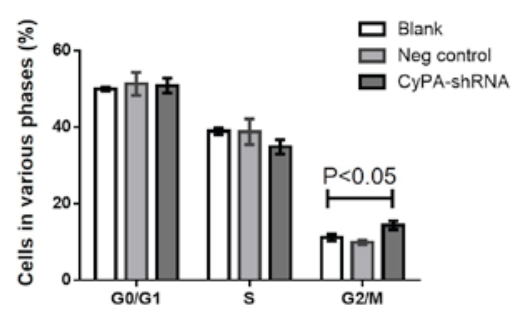

Figure 5. CyPA silencing alters the cell cycle distribution of PAa lung cancer cells without radiation. (A) Cell cycle analysis using flow cytometry. (B) Percentage of cells in various cell phases. Experiments were repeated three times and each group contained three replicates $(n=9)$. Data are presented as the mean \pm standard error. One-way analysis of variance was performed followed by Dunnett's T3 test to compare the differences among the three groups. Blank, PAa cells without transfection; Neg control, PAa cells transfected with vectors; CyPA-shRNA, PAa cells transfected with CyPA-shRNA-2. PAa, cyclophilin A-silencing lung adenocarcinoma; CyPA, cyclophilin A; shRNA, small hairpin RNA; FL2-A, fluorescence area; Neg, negative.

the CyPA gene and transduction efficiency in PAa cells, and CyPA shRNA-3 was selected.

CyPA is important during the pathogenesis of cancer, functioning as a 'molecular switch' due to its PPIase activity (18). Previous studies have demonstrated aberrant CyPA overexpression in numerous types of cancer cell (19-21). During the present study, a stable CyPA-shRNA knockdown PAa cell line was initially developed to investigate the role of CyPA gene in lung adenocarcinoma. It was observed that CyPA knockdown significantly enhanced the apoptosis of PAa cells. This is concordant with the results of a previous report, demonstrating that CyPA is an essential promoter for tumor growth (13). These findings suggest that CyPA may be a potential novel target for the treatment of lung adenocarcinoma.
A
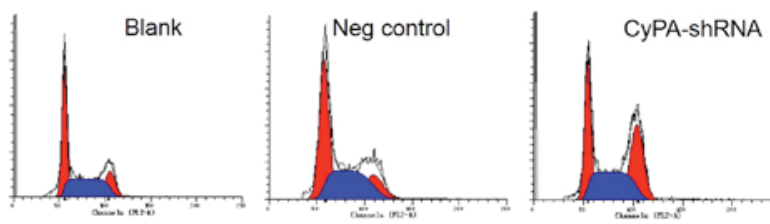

B

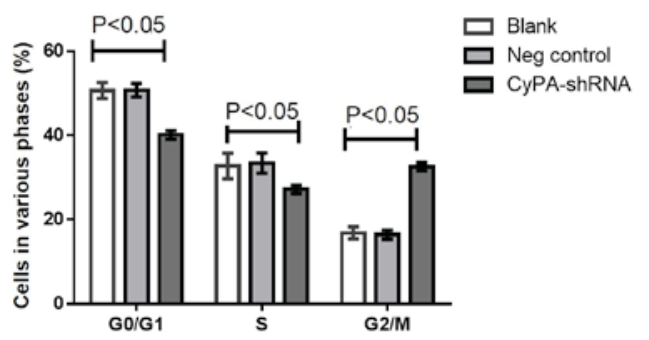

Figure 6. CyPA silencing has a more marked effect on the cell cycle distribution of PAa cells following radiation. (A) Representative cell cycle distribution analysis of PAa cells post-radiation using flow cytometry. (B) Percentage of cells in various cell cycle phases. Cells were irradiated with ${ }^{60} \mathrm{Co}$ at $2 \mathrm{~Gy}$ and subsequently cultured for a further 2 days prior to flow cytometry analysis. Experiments were repeated three times and each group included three replicates $(n=9)$. Data are presented as the mean \pm standard error of the mean. One-way analysis of variance was performed followed by Dunnett's T3 test to compare the differences among the three groups. Blank, PAa cells without transfection; Neg control, PAa cells transfected with vectors; CyPA-shRNA, PAa cells transfected with CyPA-shRNA-2. PAa, cyclophilin A-silencing lung adenocarcinoma; CyPA, cyclophilin A; shRNA, small hairpin RNA; FL2-A, fluorescence area; Neg, negative.

Radiotherapy is a common therapeutic choice for lung adenocarcinoma (4). Gene-radiotherapy, the combination of traditional radiation and targeted gene therapy is a recent breakthrough in cancer treatment (22). In a previous study, proteomic analysis of pulmonary adenocarcinoma demonstrated an upregulation of CyPA following radiotherapy (14). In the present study, the effect of CyPA knockdown on the radiosensitivity of PAa cells was investigated using fluorescence-activated cell sorting analysis and the multi-target single-hit mathematical model. It was observed that the survival fraction at 2 Gy (SF2), $\mathrm{D}_{0}$ and $\mathrm{Dq}$ decreased following CyPA-silencing. Furthermore, the Dq sensitization enhancement ratio was $>1$. It is known 
that a decrease of $\mathrm{D}_{0}$ is associated with an enhanced radiosensitizing effect, whereas a decrease in Dq is associated with weaker cellular repair following sublethal injury (23), and the SF2 value is negatively correlated with radiosensitivity (24). The aforementioned results, therefore, demonstrate that RNAi-mediated gene silencing of CyPA reduced sublethal repair injury and improved the radiosensitivity of human PAa lung adenocarcinoma cells. To the best of our knowledge, the present study is the first to demonstrate that silencing of CyPA leads to a significant increase in radiosensitivity in lung adenocarcinoma cells.

The radiosensitivity of tumor cells is closely associated with the distribution of cells within the proliferation cycle. Cells in the $G_{2} / M$ phase exhibit the highest radiosensitivity, followed by the $G_{0} / G_{1}$ phase, with the $S$ phase being the least radiosensitive stage (25). In the current study, an increase in the number of cells in the $\mathrm{G}_{2} / \mathrm{M}$ phase in the CyPA-silenced groups, compared with the blank and negative control groups, was observed. Furthermore, CyPA silencing was demonstrated to synergize with radiation, leading to marked $\mathrm{G}_{2} / \mathrm{M}$ phase arrest in PAa cells and a significant reduction in the proportion of cells in the $\mathrm{G}_{0} / \mathrm{G}_{1}$ and $\mathrm{S}$ phase. Taken together, these results indicate that CyPA silencing is able to elevate the radiosensitivity of PAa cells via $\mathrm{G}_{2} / \mathrm{M}$ phase arrest and the promotion of cell apoptosis.

A previous report demonstrated that the overexpression of CyPA in cancer cells may prevent hypoxia and anticancer drug-induced cell apoptosis through the upregulation of the extracellular signal-regulated kinase (ERK)1/2 signaling pathway (18). In addition, disruption of the ERK1/2 signaling pathway sensitizes prostate cancer cells to radiation (26). Therefore, in the present study, it is likely that he silencing of CyPA disturbed the ERK1/2 signaling cascade, thus, resulting in an increase in PAa cell radiosensitivity.

In conclusion, the current study demonstrated that lentiviral vectors are able to specifically silence the CyPA gene and increase cell apoptosis. In addition, RNAi-mediated downregulation of CyPA enhanced the radiosensitivity of lung adenocarcinoma cells, and CyPA silencing also significantly induced $\mathrm{G}_{2} / \mathrm{M}$ phase arrest. These data provide compelling evidence that the combination of CyPA gene silencing and irradiation may represent a novel potential strategy for the treatment of lung adenocarcinoma.

\section{Acknowledgements}

The present study was supported by grants from The National Key Research and Development Program of China (grant no. 2016YFC0100105), the National Science Foundation of China (grant nos. 81628008, 81571641 and 81072925) and an internal grant from the China-Japan Friendship Hospital (grant no. 2014-3-MS-18).

\section{References}

1. Siegel R, Ward E, Brawley O and Jemal A: Cancer statistics, 2011: The impact of eliminating socioeconomic and racial disparities on premature cancer deaths. CA Cancer J Clin 61: 212-236, 2011.

2. Jemal A, Center MM, DeSantis C and Ward EM: Global patterns of cancer incidence and mortality rates and trends. Cancer Epidemiol Biomarkers Prev 19: 1893-1907, 2010.
3. Houston KA, Henley SJ, Li J, White MC and Richards TB: Patterns in lung cancer incidence rates and trends by histologic type in the United States, 2004-2009. Lung Cancer 86: 22-28, 2014.

4. Baker S, Dahele M, Lagerwaard FJ and Senan S: A critical review of recent developments in radiotherapy for non-small cell lung cancer. Radiat Oncol 11: 115, 2016.

5. Gomez-Casal R, Bhattacharya C, Ganesh N, Bailey L, Basse P, Gibson M, Epperly M and Levina V: Non-small cell lung cancer cells survived ionizing radiation treatment display cancer stem cell and epithelial-mesenchymal transition phenotypes. Mol Cancer 12: 94, 2013.

6. Ma W, Ma CN, Li XD and Zhang YJ: Examining the effect of gene reduction in miR-95 and enhanced radiosensitivity in non-small cell lung cancer. Cancer Gene Ther 23: 66-71, 2016.

7. Torres-Roca JF and Stevens CW: Predicting response to clinical radiotherapy: Past, present, and future directions. Cancer Control 15: 151-156, 2008.

8. Thapar R: Roles of Prolyl Isomerases in RNA-mediated gene expression. Biomolecules 5: 974-999, 2015.

9. Nigro P, Pompilio G and Capogrossi MC: Cyclophilin A: A key player for human disease. Cell Death Dis 4: e888, 2013.

10. Feng W, Xin Y, Xiao Y, Li W and Sun D: Cyclophilin A enhances cell proliferation and xenografted tumor growth of early gastric cancer. Dig Dis Sci 60: 2700-2711, 2015.

11. Campa MJ, Wang MZ, Howard B, Fitzgerald MC and Patz EJ Jr: Protein expression profiling identifies macrophage migration inhibitory factor and cyclophilin a as potential molecular targets in non-small cell lung cancer. Cancer Res 63: 1652-1656, 2003.

12. Qian Z, Zhao X, Jiang M, Jia W, Zhang C, Wang Y, Li B and Yue W: Downregulation of cyclophilin A by siRNA diminishes non-small cell lung cancer cell growth and metastasis via the regulation of matrix metallopeptidase 9. BMC Cancer 12: 442, 2012.

13. Howard BA, Furumai R, Campa MJ, Rabbani ZN, Vujaskovic Z Wang XF and Patz EF Jr: Stable RNA interference-mediated suppression of cyclophilin A diminishes non-small-cell lung tumor growth in vivo. Cancer Res 65: 8853-8860, 2005.

14. Huang JC, Zhao PC, Zhang HZ and Wang H: A proteomical study on the radiosensitized target molecules of fuzheng zengxiao formula in pulmonary adenocarcinoma nude mice model. J Tradit Chin Med 31: 3-6, 2011.

15. Zhang S, Joseph G, Pollok K, Berthoux L, Sastry L, Luban J and Cornetta K: G2 cell cycle arrest and cyclophilin A in lentiviral gene transfer. Mol Ther 14: 546-554, 2006.

16. Dull T, Zufferey R, Kelly M, Mandel RJ, Nguyen M, Trono D and Naldini L: A third-generation lentivirus vector with a conditional packaging system. J Virol 72: 8463-8471, 1998.

17. Sampsonas F, Ryan D, McPhillips D and Breen DP: Molecular testing and personalized treatment of lung cancer. Curr Mol Pharmacol 7: 22-32, 2014

18. Brazin KN, Mallis RJ, Fulton DB and Andreotti AH: Regulation of the tyrosine kinase Itk by the peptidyl-prolyl isomerase cyclophilin A. Proc Natl Acad Sci USA 99: 1899-1904, 2002.

19. Choi KJ, Piao YJ, Lim MJ, Kim JH, Ha J, Choe W and Kim SS: Overexpressed cyclophilin A in cancer cells renders resistance to hypoxia-and cisplatin-induced cell death. Cancer Res 67: 3654-3662, 2007.

20. Li Y, Guo H, Dong D, Wu H and Li E: Expression and prognostic relevance of cyclophilin A and matrix metalloproteinase 9 in esophageal squamous cell carcinoma. Diagn Pathol 8: 207, 2013.

21. Obchoei S, Weakley SM, Wongkham S, Wongkham C, Sawanyawisuth K, Yao Q and Chen C: Cyclophilin A enhances cell proliferation and tumor growth of liver fluke-associated cholangiocarcinoma. Mol Cancer 10: 102, 2011.

22. Kaliberov SA, Kaliberova LN, Yan H, Kapoor V and Hallahan DE: Retargeted adenoviruses for radiation-guided gene delivery. Cancer Gene Ther 23: 303-314, 2016.

23. Zhuang HQ, Sun J, Yuan ZY, Wang J, Zhao LJ, Wang P, Ren XB and Wang CL: Radiosensitizing effects of gefitinib at different administration times in vitro. Cancer Sci 100: 1520-1525, 2009.

24. Björk-Eriksson T, West C, Karlsson E and Mercke C: Tumor radiosensitivity (SF2) is a prognostic factor for local control in head and neck cancers. Int J Radiat Oncol Biol Phys 46: 13-19, 2000.

25. Pawlik TM and Keyomarsi K: Role of cell cycle in mediating sensitivity to radiotherapy. Int J Radiat Oncol Biol Phys 59: 928-942, 2004

26. Wang T, Carraway RE, LaRoche D and FitzGerald TJ: Disruption of ERK1/2 Sensitizes radiation resistance prostate cancer cells to paclitaxel and ionizing radiation. Int J Radiat Oncol Biol Phys 90 (Suppl): S806, 2014. 